\title{
ANTI-TNF AND THE RISK OF TUBERCULOSIS IN WORKERS IN THE PRISON SYSTEM
}

Deivid Souza Sutti Lopes ${ }^{1, \star}$, Amanda Bernardes ${ }^{1}$, Rina Dalva Neubarth Giorgi ${ }^{1}$, Bethânia Cabral Cavalli Swiczar ${ }^{1}$, Sonia Maria Alvarenga Anti Loduca Lima ${ }^{1}$

1.Instituto de Assistência Médica ao Servidor Público Estadual, São Paulo (SP), Brazil.

*Corresponding author: deividsutti@gmail.com

\section{BACKGROUND}

Tuberculosis (TB) is one of the main causes of morbidity and mortality from infectious diseases in our country. In some situations, its prevalence is high, as in the case of prison populations and prison workers, where confinement, overcrowding, and difficulty in isolating infected patients make the environment favorable to transmission of Mycobacterium tuberculosis. In rheumatology clinics it is common to use anti-TNFa immunobiological drugs to control autoimmune conditions such as spondyloarthritis, psoriatic arthritis, among others. These drugs are known to increase the risk of developing TB, since TNFa is essential for the formation and maintenance of granulomas, therefore its inhibition may increase the risk of developing or reactivating latent infection. We will report two cases of patients, prison workers, with spondyloarthritis who developed TB during anti-TNF therapy, even with negative screening tests (PPD and chest X-ray) and do not using concomitant glucocorticoids.

\section{CASE REPORT}

Patient 1: male, 43 years old, prison guard, diagnosed with psoriatic arthritis since 1997, developed peritoneal TB after 3 years of adalimumab monotherapy. The condition started with abdominal pain, fever and night sweats. A biopsy of the intra-abdominal lesions was necessary to close the diagnosis. Patient 2: male, 67 years old, prison guard, diagnosed with spondyloarthritis since 2012. He developed pulmonary TB after 6 years of adalimumab use. At TB diagnosis, he was taking concomitant sulfasalazine. The condition started with cough, afternoon fever and weight loss. The diagnosis was based on symptoms and complementary tests such as chest X-ray and PPD. Both patients needed to discontinue anti-TNFa, treat tuberculosis, and then switch the medication's mechanism of action to treat the underlying pathologies, in order to reduce the risk of reactivation of the infectious disease.

\section{CONCLUSION}

The report of these cases reminds us of the need for special care in monitoring patients taking anti-TNF drugs who are prison workers, and may also indicate that, in this group of professionals, the choice of immunobiologicals with mechanisms of action other than TNFa inhibition is more appropriate to minimize the risk of TB.

\section{KEYWORDS:}

Tuberculosis, Anti-TNFa, Prison system. 